\title{
A importância da nutrição pós-cirúrgica no paciente pediátrico: uma revisão narrativa
}

\author{
The importance of post-surgical nutrition in pediatric patients: a narrative review
}

La importancia de la nutrición posquirúrgica en pacientes pediátricos: una revisión narrativa

Isabela Oliveira Eugenio ${ }^{1 *}$, Estéfany Kotaka Munhoz ${ }^{2}$, Daniela Batista Souza ${ }^{1}$, Petrus Ariel Vieira dos Santos ${ }^{3}$, Príncia Christino de Abreu Carvalho ${ }^{1}$, Erick Lessa Ramos ${ }^{4}$, Marcela Annechino Novais $^{4}$, Wagner Pablo Corrêa ${ }^{5}$.

\section{RESUMO}

Objetivo: Revisar e demonstrar, por meio de uma revisão narrativa, a importância da nutrição pediátrica em pacientes submetidos a cirurgias. Revisão Bibliográfica: A mortalidade infantil associada à cirurgia decresceu rapidamente devido à melhoria da assistência nutricional a esses pacientes. Sabe-se que, em suma, a maioria dos procedimentos cirúrgicos na pediatria ocorre em nível ambulatorial, assim, há menor permanência no centro hospitalar, deambulação precoce e rápida recuperação. Porém, uma criança má nutrida pode evoluir para complicações indesejadas. É necessário a escolha do método alimentar mais adequado para cada paciente e também de sua via mais apropriada, podendo ser por via oral espontânea, por meio de sondas de longa duração como as nasogástricas e nasoenterais, assim como por gastrostomias e jejunostomias. Considerações finais: É necessário o médico compreender as necessidades nutricionais de cada paciente, respeitando sua idade e particularidades, para, assim, providenciar as devidas demandas metabólicas e os nutrientes necessários, reduzindo o risco de complicações no pós-operatório.

Palavras-chave: Nutrição da criança, Recuperação pós-cirúrgica melhorada, Metabolismo.

\section{ABSTRACT}

Objective: To review and demonstrate, through a narrative review, the importance of pediatric nutrition in surgical patients. Literature review: Infant mortality associated with surgery decreased rapidly due to improved nutritional assistance for these patients. It is known that, in short, most surgical procedures in pediatrics occur on an outpatient basis, thus, there is less hospital stay, early ambulation and quick recovery. However, a malnourished child can progress to unwanted complications. It is necessary to choose the most adequate food method for each patient and also its most appropriate route, which can be spontaneously orally, through long-term tubes such as nasogastric and nasoenteral tubes, as well as gastrostomies and jejunostomies. Final considerations: It is necessary for the physician to know the nutritional needs of each patient, respecting their age and particularities, in order to provide the necessary metabolic demands and the necessary nutrients, before the risk of complications in the postoperative period.

Key words: Child nutrition, Enhanced recovery after surgery, Metabolism.

\footnotetext{
1 União Educacional do Vale do Aço (UNIVAÇO), Ipatinga - MG. *E-mail: isabelaoliveira.e@gmail.com

${ }^{2}$ Fundação Educacional do Município de Assis (FEMA), Assis - SP.

3 Universidade Nove de Julho (UNINOVE), São Paulo - SP.

${ }^{4}$ Centro Universitário de Volta Redonda (UniFOA), Volta Redonda - RJ.

${ }^{5}$ Centro Universitário de Caratinga, Caratinga - MG.
}

SUBMETIDO EM: 6/2021

ACEITO EM: 6/2021

PUBLICADO EM: 7/2021 


\section{RESUMEN}

Objetivo: Revisar y demostrar, a través de una revisión de la literatura, la importancia de la nutrición pediátrica en pacientes sometidos a cirugía. Revisión bibliográfica: La mortalidad infantil asociada con la cirugía disminuyó rápidamente debido a la mejor atención nutricional de estos pacientes. Se sabe que, en definitiva, la mayoría de los procedimientos quirúrgicos en pediatría se realizan de forma ambulatoria, por lo que hay menos estancia en el hospital, caminata temprana y recuperación rápida. Sin embargo, un niño mal alimentado puede progresar a complicaciones no deseadas. Es necesario elegir el método dietético más adecuado para cada paciente y también la vía más adecuada, que puede ser de forma espontánea por vía oral, a través de sondas de larga duración como nasogástricas y nasoenterales, así como gastrostomías y yeyunostomías. Consideraciones finales: Es necesario que el médico comprenda las necesidades nutricionales de cada paciente, respetando su edad y particularidades, con el fin de aportar las demandas metabólicas necesarias y los nutrientes necesarios, reduciendo el riesgo de complicaciones en el postoperatorio.

Palabras clave: Nutrición del niño, Recuperación mejorada después de la cirugía, Metabolismo.

\section{INTRODUÇÃO}

Historicamente, a mortalidade infantil associada à cirurgia decresceu rapidamente, já que as mudanças no período perioperatório estão mais compreensíveis. Essas mudanças geradas por traumas cirúrgicos, que podem ser metabólicas, fisiológicas e patológicas, têm sido mais claras para toda a equipe multidisciplinar da área da saúde, devido a diversos estudos que os capacitaram analisar parâmetros vitais do corpo humano, em especial, os de pacientes pediátricos. A nutrição adequada individualizada pediátrica pós-cirúrgica ganhou destaque no meio científico, bem como o suporte nutricional nos últimos anos (CORADINE AVP, et al., 2015). A alimentação enteral é a primeira opção para a terapia nutricional, porém, caso o trato gastrointestinal esteja comprometido, a Nutrição Parenteral (NP) é indicada (FALCÃO MC e TANNURI U, 2020).

Com relação à NP dos pacientes pediátricos, as formulações são, normalmente, individualizadas devido às necessidades relacionadas ao seu crescimento e desenvolvimento e, consequentemente, são demandas nutricionais diferentes. O maior benefício dessa é a indicação precisa de acordo com as necessidades nutricionais e a condição clínica de cada indivíduo. Dessa forma, para uma dieta adequada e segura, é preciso saber as necessidades de proteína, energia, macronutrientes, micronutrientes e equilíbrio ácido base feito por profissionais capacitados, auxiliados pelas farmácias de manipulação para tal nutrição (FREITAS RGBON, et al., 2014)

A desnutrição está relacionada a um mal prognóstico no paciente hospitalizado, visto que possui ligação com o aumento do risco de infecções, perda de massa muscular, prejuízo na cicatrização de feridas, maior tempo de internação e aumento da morbimortalidade. Em pacientes pediátricos, ainda pode haver prejuízo no crescimento e no desenvolvimento cognitivo, assim como baixo desempenho escolar (COSTA CAD, et al., 2018). Assim, o foco dos profissionais é a manutenção do peso corporal, o que evita que os pacientes sofram desnutrição durante a internação (OLIVEIRA AF, et al., 2005).

O presente estudo teve como objetivo avaliar a importância da nutrição na recuperação e desenvolvimento de pacientes pediátricos no pós-operatório, uma vez que tal aporte nutricional, feito de forma adequada, promove e acelera o processo de recuperação, além de diminuir os riscos de infecções por depleção do sistema imunológico causado pela desnutrição.

\section{REVISÃO BIBLIOGRÁFICA}

As cirurgias pediátricas são, em sua maioria, realizadas em nível ambulatorial, pois assim necessitam de menor tempo de internação (quando necessitam) e de cuidados pós-operatórios menos intensivos. Assim, os pacientes ficam menos expostos ao ambiente hospitalar. Contudo, a cirurgia é considerada um evento traumático físico e emocional para a criança, gerando emoções como ansiedade, que pode acarretar 
alterações nos sinais vitais, como o aumento da pressão arterial, aumento das frequências cardíaca e respiratória, além de aumentar o consumo de oxigênio e ocasionar tensão muscular (FRANZOI MAH e MARTINS G, 2016; BURDEN S, et al., 2012).

O procedimento cirúrgico é considerado e encarado como um trauma para as pacientes pediátricos, principalmente pelo fato de que em geral esses pacientes nunca passaram por cirurgias prévias e não possuem compreensão adequada do procedimento. Além disso, a realização da cirurgia causa afastamento temporário de seus familiares e de suas atividades habituais. Muitos pacientes também sentem medo da dor que pode ocorrer no período Pós-Operatório (PO), assim como medo da anestesia. Esse medo em geral acarreta ansiedade na criança, que tem como consequências agressividade, agitação, alteração dos sinais vitais, má qualidade de sono, assim como resposta inadequada à anestesia (FRANZOI MAH e MARTINS G, 2016).

Em muitos casos, a angústia com a realização da cirurgia tem origem nos pais e familiares da criança, que se sentem preocupados e inseguros e muitas vezes acabam por transmitir tais sentimentos aos pacientes pediátricos. A insegurança dos familiares em geral ocorre por medo do procedimento e medo da anestesia e muitas vezes ocorre por falta de informação (FRANZOI MAH e MARTINS G, 2016).

Uma cirurgia causa resposta nos sistemas metabólico, imunológico e endócrino do indivíduo, pois o estresse cirúrgico realiza estimulação neuronal eferente, que ativa vias imunes celulares e humorais, gerando reações inflamatórias e aumento de citocinas, como, por exemplo, interleucina 6 (IL-6) e fator de necrose tumoral a (TNF- $\alpha$ ), que além de ser citocina pró inflamatória, é mediador precoce da neuroinflamação e da sensibilização de dor central. Uma cirurgia também possui um alto efeito catabólico sobre os sistemas do paciente, o que pode ser agravado caso o estado nutricional prévio da criança não seja adequado (SOUZA NA, et al., 2019).

Durante o trauma, a resposta do paciente ao recebimento de nutrientes é diferente da resposta encontrada em um paciente que não sofreu lesão, estando apenas sob efeito de jejum, tendo a oferta de calorias vindo $90 \%$ de depósitos de lipídios e apenas 5 a $8 \%$ derivadas das proteínas. Como discutido, na resposta ao trauma, cerca de $30 \%$ das calorias são provenientes de proteínas endógenas e somente $50 \%$ vem das gorduras. O resultado do estímulo hormonal do estresse advindo do trauma resulta em consumo proteico para produção de energia, que acarreta em gliconeogênese com consequente produção de glicose, que tem como fonte os aminoácidos, que no fígado sofrem conversão em alanina e, por ultimo, glicose (MEDEIROS AC e FILHO AMD, 2017).

Além dessas alterações, o período em que uma criança saudável permanece em jejum antes da cirurgia também pode alterar sua condição metabólica. Ocorre maior acúmulo de corpos cetônicos no sangue quanto maior é o tempo de jejum, que por sua vez, possui caráter catabólico, elevando a sua resposta metabólica ao trauma. Da mesma forma, o jejum prolongado também pode provocar fome e sede, irritabilidade, ansiedade, desconforto, mal-estar, desidratação (podendo provocar dificuldades na realização de acesso venoso), cefaleia, atraso na recuperação cirúrgica, hipoglicemia e tempo prolongado para despertar da anestesia (CARVALHO CALB, et al., 2017).

Os pacientes pediátricos com longo tempo de jejum pré-operatório, em geral, também apresentam diminuição das reservas de glicogênio e aumento da gliconeogênese, assim como piora do catabolismo, com aumento de ácidos graxos e corpos cetônicos, além de maior hiperglicemia e resistência à insulina no período que sucede a cirurgia (CARVALHO CALB, et al., 2020).

Existe uma relação entre a gravidade e extensão do trauma com a taxa de hipoinsulinemia do paciente. Em consequência, ocorre aumento da insulina de forma muito baixa em relação à glicemia. Ocorre, então, aumento da frequência de excreção urinária, inferindo maior ou menor perda de insulina degradada pelos rins, que tem, no período pós-traumático, a meia vida reduzida (MEDEIROS AC e FILHO AMD, 2017).

Historicamente, após estudos realizados pelos grupos Enhanced Recovery After Surgery (ERAS (Europeu) e American Society of Anaesthesiologists (ASA)(Americano) ocorreu à implantação do protocolo de Aceleração da Recuperação Total Pós-Operatória, denominado ACERTO, onde o tempo de jejum para sólidos 
foi mantido, mas os pacientes passaram a tomar duas horas antes do procedimento cirúrgico uma bebida com volume de $200 \mathrm{ml}$ acrescida de maltodextrina a 12\% (LUDWIG RB, et al., 2013).

Com o intuito de reduzir o tempo prolongado do jejum pré-operatório pode- se utilizar bebidas ricas em carboidratos, sendo administradas em até duas horas antes do procedimento cirúrgico. Alguns estudos vêm relatando os benefícios desta conduta para o melhor prognóstico do paciente, além disso, auxilia no bemestar, melhora o metabolismo glicêmico, diminuindo assim a resistência insulínica em até $50 \%$ no pósoperatório o que propicia a redução da perda de massa magra, dessa forma, favorecendo um melhor resultado no PO, favorecendo o processo de recuperação (CARVALHO CALB, et al.,2020).

Tal evolução é de extrema relevância uma vez que anualmente são realizados cerca de 234 milhões de procedimentos cirúrgicos por ano no mundo, visto que é uma opção de tratamento comum e eficaz para diversas doenças. Nesse viés, as complicações pós-operatórias decorrentes de operações de grande porte continuam a ser uma das principais causas da morbidade e mortalidade dos procedimentos. Dessa maneira, é recomendado que os profissionais da saúde abandonem as antigas indicações de longos períodos de jejum devido às novas evidências para orientação das práticas pré-anestésicas e os aspectos negativos que devem ser considerados, como: pacientes permanecem muito mais tempo de jejum que o estabelecido, por várias razões como, atraso nas operações, transferência de horário ou local de realização do procedimento. Logo, cabe à equipe multidisciplinar encorajar-se para se ajustar aos novos protocolos de jejum pré-operatório para proporcionar mais benefícios aos pacientes (LUDWIG RB, et al., 2013).

Pacientes pediátricos e lactentes são mais suscetíveis a sofrerem ações do estresse metabólico. $O$ estado nutricional insuficiente leva a uma perda do tecido adiposo e muscular, que pode agravar a desnutrição, e assim, favorecer a instalação de processos infecciosos. Contudo, a indicação específica de um suporte nutricional parenteral individualizada melhora o estado nutricional e a condição clínica dos pacientes, visto que ocorre a adequação energética proteica, uma diminuição dos riscos de infecção e uma melhora metabólica (FREITAS RGBON, et al., 2014).

Alterações no metabolismo dos lipídeos, carboidratos, proteínas, micronutrientes e balanço eletrolítico decorrentes do estresse metabólico que compromete o sistema imune e facilita a instalação de infecções, podem acarretar em desnutrição aguda, síndrome de disfunção múltipla de órgãos e morte. Visto isso, a nutrição individualizada deve ocorrer através da manutenção do peso corporal, adequação dos macronutrientes e micronutrientes a fim de evitar que o paciente sofra depleção e consequentemente melhore o estado nutricional (OLIVEIRA AF, et al., 2005).

A terapêutica nutricional é bastante variável e depende da doença de base, tendo a avaliação nutricional como fator preponderante para a adequação da dieta. A avaliação nutricional permite que o paciente seja identificado quanto ao risco nutricional durante a admissão e internação, possibilitando a intervenção precoce no pré-operatório e a adequação da dieta no pós-operatório, permitindo um melhor controle do tratamento e recuperação do estado (SIMÕES AP, et al., 2010).

A necessidade energética e nutricional de cada indivíduo é definida como a quantidade mínima de nutrientes necessários para garantir o bom funcionamento orgânico e de suas funções fisiológicas (SAMPAIO AS, et al., 2018; JOFFE A, et al., 2016). Esta, por sua vez, se relaciona diretamente à faixa etária, sexo, reservas corpóreas, estado nutricional, risco nutricional e estado metabólico do paciente (CORDEIRO MLB, et al., 2019).

A manutenção de uma alimentação adequada é de suma importância, visto que as crianças apresentam imaturidade de suas funções orgânicas e imunológicas (MOURA MAP, et al., 2015). Desse modo, é notável que qualquer alteração nutricional é capaz de interferir negativamente no crescimento e desenvolvimento infantil. O uso do método de nutrição secundária, enteral ou parenteral, tem sido uma estratégia importante e necessária no aperfeiçoamento do suporte nutricional em pacientes pediátricos pós-cirúrgicos que não conseguem obter sua nutrição por via oral no PO (CORDEIRO MLB, et al., 2019).

A nutrição enteral é uma opção de dieta realizada por meio de uma sonda inserida no estômago, duodeno ou jejuno e está indicada em casos de risco de aspiração pulmonar, em pacientes com distúrbio de deglutição 
que possam sofrer uma aspiração pulmonar, em pacientes críticos em ventilação mecânica, alteração do nível de consciência, impossibilidade ter as necessidades de energia e proteína somente por via oral, em estados hipercatabólicos como sepses e queimaduras graves, em recém nascidos menores de 34 semanas sem capacidade de alimentação oral, na transição da NP para oral, em casos de obstrução do trato digestivo alto e realimentação do paciente desnutrido. As principais contraindicações são a obstrução e a semi obstrução intestinais e a instabilidade hemodinâmica. As demais são relativas e dependem de cada indivíduo (FERNANDES VPI, et al., 2013).

A administração da nutrição enteral para crianças é semelhante a dos adultos, podendo ser administrado de forma intermitente ou contínua, com ou sem repouso à noite. Portanto, sua forma dependerá da tolerância, modo de administração e comorbidades de cada paciente. Além disso, para nutrição enteral intermitente, cuidados especiais para bebês com baixo peso ao nascer são essenciais, pois se a velocidade do tratamento nutricional não for supervisionada por um profissional bem treinado, há risco de alterações respiratórias (FERNANDES VPI, et al., 2013).

Vale ressaltar que, para a realização da nutrição enteral, são utilizados dois tipos de sonda, as quais se diferenciam pelo tempo de permanência, isto é, de longa duração (nasogástricas e nasoenterais) e curta duração (gastrostomias e jejunostomias) (FERNANDES VPI, et al., 2013). Quando contraindicada, deve-se priorizar o uso da (NP), que está indicado em condições de: pré-operatório e pós-operatório, insuficiência hepática com desnutrição, insuficiência renal com desnutrição, doenças gastrointestinais, traumas e queimaduras, malformações congênitas, prematuridade e enterocolite necrosante (SILVA SLC, et al., 2014).

A NP é uma estratégia nutricional intravenosa que ultrapassa o Trato Gastrointestinal (TGI), utilizada com o intuito de fornecer nutrientes necessários para manter o metabolismo ou restituir o organismo, garantindo um desenvolvimento ideal (MELRO EC, 2019). Em relação às vias de administração, a NP pode utilizar da via central ou periférica. Diante disso, a escolha sobre qual paciente deve ser submetido à NP e sobre a principal via a ser utilizada, se relaciona com a doença de base do paciente e a experiência da equipe médica (TÜTÜNCÜ AÇ, et al., 2013).

Nessa perspectiva, é importante que seja realizada uma avaliação do balanço hídrico de forma individualizada por meio da análise da massa corpórea, faixa etária e condições clínicas do paciente. Para isso, a fim de determinar as necessidades hídricas do pediátrico, tem-se como alternativa lançar mão da fórmula de Holliday Segar ou o cálculo da superfície corporal (SILVA SLC, et al., 2014).

Além disso, deve-se também estimar a necessidade energética de cada paciente antes do início da NP. Contudo, trata-se de um manejo complexo, visto que essa sofre influência do metabolismo e do estado clínico. Portanto, para uma melhor nutrição utiliza-se como base o gasto energético basal somado ao gasto energético funcional da criança (MELRO EC, 2019).

A NP é considerada um suporte nutricional eficaz para sobrevida e reabilitação de pacientes pediátricos e está associada a complicações como infecções, hipertrigliceridemia, distúrbios metabólicos, alterações hepáticas e distúrbios de minerais. Dessa forma, deve-se priorizar seu uso em situações temporárias, visando a recuperação do paciente e a suspensão desta estratégia assim que reinstituída sua capacidade de nutrição por via oral ou enteral (MELRO EC, 2019).

A desnutrição favorece a instalação de processos infecciosos, visto que compromete o funcionamento do sistema imunológico, além de comprometer o estado nutricional do paciente. Infecções que acometem o trato gastrointestinal são capazes de alterarem a absorção e biodisponibilidade dos nutrientes, podendo levar a um desbalanço eletrolítico, ao aumento na glicogênese e lipogênese, interferindo no metabolismo de carboidratos, lipídeos, proteínas e níveis de micronutrientes (OLIVEIRA AF, et al., 2005).

A desnutrição está diretamente relacionada ao pior prognóstico em pacientes hospitalizados, principalmente nos pacientes pediátricos, acarretando geralmente maior tempo de permanência no hospital, prolongando o tempo de cicatrização, diminuindo a quantidade de massa magra do paciente e interferindo, até mesmo, no crescimento e desenvolvimento da criança. Isso mostra a importância da rápida identificação da desnutrição e sua consequente correção (OLIVEIRA TC, et al., 2017). Com isso, o acompanhamento 
nutricional pós-cirúrgico adequado visa diminuir os transtornos que ocorrem durante o procedimento, assim como diminuir os riscos de desnutrição, restabelecer a homeostase metabólica e proporcionar uma recuperação mais rápida do paciente (CAVALCANTE NCF, et al., 2017).

\section{CONSIDERAÇÕES FINAIS}

A nutrição pós-cirúrgica na pediatria é vital para a melhor recuperação do paciente. Sendo assim, é necessário escolher a melhor dieta de forma individualizada avaliando também sua via de administração e o tipo de sonda a ser escolhida, Além disso, para estabelecer um melhor prognóstico é imperioso se atentar às alterações metabólicas a fim de mitigar possíveis complicações. Por fim, a escolha adequada do método nutricional vai interferir desde a alta hospitalar precoce até a melhor cicatrização do ferimento cirúrgico proporcionando assim maior bem-estar ao paciente.

\section{REFERÊNCIAS}

1. BURDEN S, et al. Pre-operative Nutrition Support in Patients Undergoing Gastrointestinal Surgery. Cochrane Database of Systematic Reviews, 2012; 11: 1-54.

2. CARVALHO CALB, et al. Mudando paradigmas em jejum pré-operatório: resultados de um mutirão em cirurgia pediátrica. ABCD Arq Bras Cir Dig, 2017; 30(1): 7-10.

3. CARVALHO CALB, et al. Benefícios Metabólicos e Inflamatórios da Abreviação do Jejum Pré-operatório em Cirurgia Pediátrica. Rev Col Bras Cir., 2020; 47: 1-10.

4. CAVALCANTE NCF, et al. Acompanhamento nutricional de um paciente pediátrico no pós-operatório da correção da Tetralogia de Fallot: estudo de caso. Arq. Catarin Med., 2017; 46(4): 154-161.

5. CORADINE AVP, et al. Medidas antropométricas para o acompanhamento do estado nutricional de crianças e adolescentes com câncer, o que utilizar na prática clínica? Rev. Bras. Cancerol, 2015; 61(3): 269-276.

6. CORDEIRO MLB, et al. Nutrição Enteral Domiciliar (NED) em crianças e adolescentes. Rev Chil Pediatr., 2019; 90(2): 222-228.

7. COSTA CAD, et al. Redução da desnutrição em pacientes pediátricos gravemente enfermos. Rev. bras. ter. intensiva, 2018; 30(2): 160-165.

8. FALCÃO MC, TANNURI U. Nutrition for the pediatric surgical patient: approach in the peri-operativa period. Rev. Hosp. Clín. Fac. Med, 2020; 57(6): 299-308.

9. FERNANDES VPI, et al. Nutrição enteral em pediatria. Residência Pediátrica, 2013; 3(3): 67-75.

10. FRANZOI MAH, MARTINS G, 2016. Ansiedade de crianças em situação cirúrgica e percepções emocionais reportadas por seus acompanhantes no pré-operatório: um estudo exploratório. REME Rev. Min. Enferm., 2016; 20: 984.

11. FREITAS RGBON, et al. Deve-se individualizar a nutrição parenteral pediátrica? Revista Paulista de Pediatria, 2014; 32(4): 326-332.

12. JOFFE A, et al. Nutritional support for critically ill children. Cochrane Database of Systematic Reviews, 2016; 5: 1-31.

13. LUDWIG RB, et al. Menor tempo de jejum pré-operatório e alimentação precoce no pós-operatório são seguros? ABCD Arq Bras Cir Dig, 2013; 26(1): 54-58.

14. MEDEIROS AC, FILHO AMD. Resposta metabólica ao trauma. J Surg CI Res, 2017; 8(1): 56-76.

15. MELRO EC. Avaliação da terapia de nutrição enteral no paciente pediátrico criticamente enfermo. Dissertação (Mestrado) - Universidade Estadual de Campinas, Faculdade de Ciências Médicas, Campinas, São Paulo, 2019; $113 p$.

16. MOURA MAP, et al. Facilidades e dificuldades dos enfermeiros no cuidar da alimentação infantil na atenção básica. Mundo saúde, 2015; 39(2): 231-238.

17. OLIVEIRA AF, et al. Evolução nutricional de crianças hospitalizadas e sob acompanhamento nutricional. Revista de Nutrição, 2005; 18(3): 341-348.

18. OLIVEIRA TC, et al. Estado nutricional de crianças e adolescentes hospitalizados: comparação entre duas ferramentas de avaliação nutricional com parâmetros antropométricos. Rev. paul. pediatr, 2017; 35(3): 273-280.

19. SAMPAIO AS, et al. Evidências sobre técnicas e parâmetros de avaliação nutricional utilizados para determinar 0 estado nutricional de crianças e adolescentes: revisão sistemática. Ciência Saúde Coletiva, 2018; 23(12): 1-12.

20. SILVA SLC, et al. Nutrição parenteral em Pediatria: revisão de literatura. Rev Med Minas Gerais, $2014 ; 24$ (Supl 2): S66-S74.

21. SOUZA NA, et al. Dislipidemia familiar e fatores associados a alterações no perfil lipídico em crianças. Ciência Saúde Coletiva, 2019; 24(1): 1-10.

22. TÛTÛNCÛ AÇ, et al. Effects of Preemptive Epidural Infusion on Cytokine Response and Postoperative Pain in Pediatric Patients. Haseki Tıp Bülteni, 2013; 51(4):162-167. 